\title{
Optical characterization of porous alumina from vacuum ultraviolet to midinfrared
}

\author{
Daniel W. Thompson, a) Paul G. Snyder, Leon Castro, Li Yan, \\ Prasuna Kaipa, and John A. Woollam \\ Department of Electrical Engineering, University of Nebraska-Lincoln, Lincoln, Nebraska 68588-0511
}

(Received 20 September 2004; accepted 31 March 2005; published online 26 May 2005)

\begin{abstract}
Porous alumina was fabricated and optically characterized over a wide spectral range. Layers were formed electrochemically in oxalic acid solution from $10-\mu \mathrm{m}$-thick aluminum films evaporated onto silicon wafers. The layer formation was monitored with in situ spectroscopic ellipsometry in the visible and near-infrared wavelength range to accurately determine the thickness and dielectric functions. Anisotropy due to the columnar nature of the porous structure was determined using optical modeling. The porous alumina layer was found to have a small but significant absorption tail throughout the visible region. Atomic force microscopy and scanning electron microscopy were used throughout the process to assess the quality of pore formation. The mean pore center-to-center spacing was approximately $100 \mathrm{~nm}$ with thicknesses up to $5 \mu \mathrm{m}$. The infrared spectra revealed absorption peaks previously seen in ceramic alumina and peaks not associated with bulk alumina. (C) 2005 American Institute of Physics. [DOI: 10.1063/1.1921336]
\end{abstract}

\section{INTRODUCTION}

Porous materials such as silicon, silica, and alumina have applications in quantum devices, optical devices, and biosensors. Optical characterization of these materials can provide information on their nanostructural properties, especially pore volume fraction $r$ and shape (e.g., cylindrical, ellipsoidal, or spherical). Since their dimensions (pore size and spacing) are often considerably smaller than the wavelengths of even ultraviolet radiation, they can be modeled as effective optical media with properties which depend on $r$ and shape, but largely independent of feature size.

While porous silicon has been studied more extensively, the fabrication of porous alumina with much more ordered nanostructure has become more common in recent years. ${ }^{1-4}$ This ordered nature is especially beneficial for use in nanotechnology, and is potentially useful in biosensing as well. The enhancement of biochemical signatures in the infrared has been demonstrated by surface area enhancement in porous silicon. ${ }^{5-11}$ Ex situ optical characterization of porous alumina has been performed in the visible range using ellipsometry, ${ }^{12,13}$ and oligonucleotide adsorption to functionalized porous alumina has been monitored in real time with optical reflectivity. ${ }^{14}$ Porous alumina for biosensing could have the advantage of a simpler optical model than is necessary with porous silicon, as well as more ordered surface area enhancement. The analyses of in situ spectroscopic ellipsometry (SE) and ex situ midinfrared (mid-IR) SE measurements described here provide further insight into optical modeling of nanoporous alumina. While the methods should be applicable to other studies, the specific optical and structural characteristics determined will be functions of process conditions.

\footnotetext{
a) Author to whom correspondence should be addressed; electronic mail: dthompson2@unl.edu
}

\section{EXPERIMENT}

To provide optical access, a 100-mm diameter cylindrical glass vessel was fitted with quartz windows and annealed for stress relief to minimize window birefringence. The windows intruded into the vessel to reduce the optical path through solution to approximately $50 \mathrm{~mm}$. The vessel input window was aligned to the light source by inspection of the back-reflected beam. Since the vessel was mounted on the $\theta$ stage of a $\theta-2 \theta$ goniometer, the vessel could be rotated precisely and tilted to direct the back reflection of the output window to the light source. The angle of separation between the windows was measured in this way to be $144.2^{\circ}$. Prealignment of the windows reduced angle uncertainties in subsequent measurements.

Samples were suspended above the cell on a precision rotation stage, readable to 5 arc min, mounted on a heavyduty optical post. The top of the sample was clamped between a stainless-steel plate (anode) and a polyethylene mount which provided electrical isolation from its tilt stage. The sample was oriented toward the light source and aligned by inspection of its back reflection, which was significantly more intense than that of the input window. The sample was rotated $72^{\circ} 5^{\prime}$ and the output beam directed into the detector (mounted on the $2 \theta$ stage) by translating the sample in the direction of normal using a stage which connected the rotation stage to the tilt mount. The alignment method was tested with a thermally grown oxide layer of known thickness (26.0 $\mathrm{nm}$ ) on silicon. Since the optical properties of silicon and its oxide are well known, the angle of incidence could be determined experimentally and was found to be within $0.1^{\circ}$ of the nominal value. The effect of window birefringence can be assessed independently in this case, ${ }^{15}$ and was found to be negligible.

Silicon wafers coated by electron-beam evaporation with a 10-nm titanium adhesion layer and $10 \mu \mathrm{m}$ of aluminum were purchased from Silicon Valley Microelectronics. The 


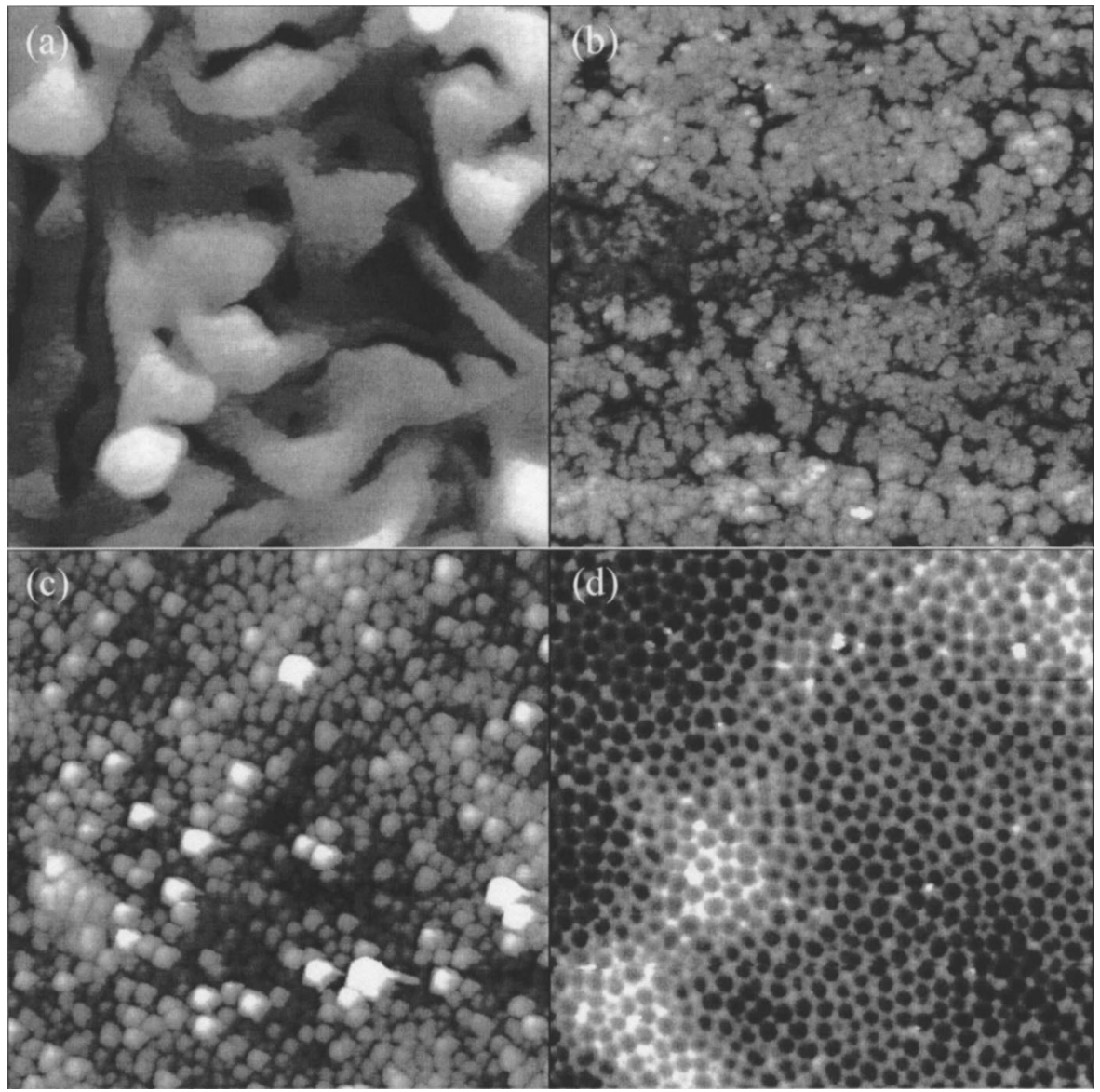

FIG. 1. Atomic force micrographs of the various stages of the process (vertical range, $\mathrm{nm}$ ): (a) As-evaporated aluminum [50], (b) after the first anodization [25], (c) after the first oxide strip [45], and (d) after the second anodization [70]. Each image is a $2-\mu \mathrm{m}$ square.

as-deposited surfaces were not nanoscopically smooth, as shown by atomic force microscopy (AFM) in Fig. 1. After the first anodization step, the surface retained a relatively rough surface layer. This sacrificial oxide layer was chemically stripped in a chromic/phosphoric acid solution $(1.25 \% / 2.12 \% \mathrm{v} / \mathrm{v}$ in de-ionized water), requiring about 4 $\mathrm{h}$ for a $1-\mu \mathrm{m}$-thick porous layer. Subsequently grown porous layers exhibited similar characteristics, with a significant reduction in surface roughness. The various surface characteristics at different stages of the process are also shown in the figure. Figure 1(d) shows the beginning of self-ordered pore formation. Others have demonstrated methods for increasing the order of pores in alumina, such as surface pretreatment ${ }^{1}$ and thicker sacrificial layers. ${ }^{16}$ The thickness of the sacrificial layer in our study was limited by the starting aluminum thickness.

Porous layers were fabricated by anodic deposition in $3 \%$ oxalic acid using a 304 stainless-steel cathode. Two coats of high-vacuum curable sealant (Vacseal, Space Environment Laboratories) were applied to coat the sample on all silicon surfaces, since silicon also anodizes in the oxalic acid solution. The aluminum was masked with enamel paint to define the active area and eliminate edge effects at the surface of the solution. Typical active areas were rectangular, about 2 $\times 1 \mathrm{~cm}^{2}$ or larger.

To encourage a linear growth rate, the current density was held constant at $6.37 \mathrm{~mA} / \mathrm{cm}^{2}$, a quantity used in another work. ${ }^{3}$ Typical time dependences of anodization voltage are shown in Fig. 2. The stages of growth have been described by Menon ${ }^{2}$ for a constant voltage process and summarized here conversely for a constant current process. The early portion of the anodization requires increasing voltage, which is dropped across the thickening barrier layer of continuous alumina. The voltage peaks when pores begin forming, followed by a minimum when the pores reach their maximum diameter. The voltage reaches steady state when the oxidation rate at the $\mathrm{Al} / \mathrm{Al}_{2} \mathrm{O}_{3}$ interface equals the pore formation rate, resulting in a barrier layer of constant thickness. With fresh solution, the steady-state voltage is about 40 $\mathrm{V}$; as the solution ages, the same current density requires more voltage and the growth rate is reduced proportionally. A subsequent anodization voltage plot also shows less pronounced curvature, demonstrating that the process of layer formation is partially accomplished $a$ priori.

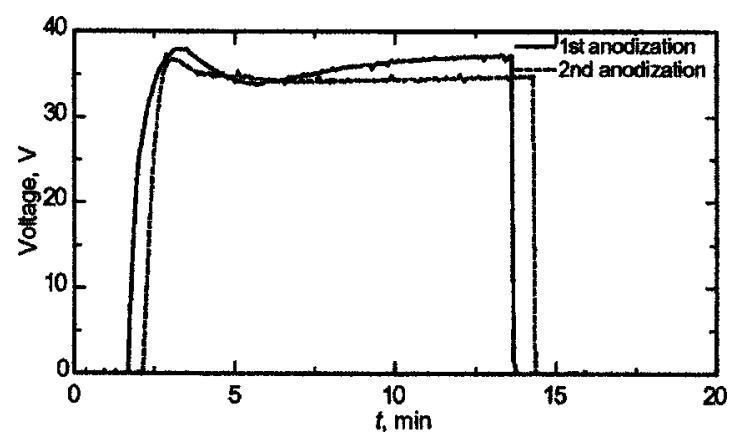

FIG. 2. Anodization voltage as a function of time for the initial surface and previously anodized and stripped surface. 


\section{OPTICAL DISPERSION MODELS AND REGRESSION FITTING}

The ellipsometric parameters $\psi$ and $\Delta$ (Ref. 17) are a measure of the optical polarization state response of a sample. Theoretical values for $\psi$ and $\Delta$ can be calculated from a mathematical model with initial estimates of thickness and dielectric function $\varepsilon(E)$ for each layer. These may be varied as parameters using a Levenberg-Marquardt algorithm to minimize the mean-squared error ${ }^{15,18}$ (MSE) between model and experiment:

$$
\mathrm{MSE}=\frac{1}{2 N-M} \sum_{i=1}^{N}\left[\left(\frac{\psi_{i}^{\bmod }-\psi_{i}^{\exp }}{\sigma_{\psi, i}^{\exp }}\right)^{2}+\left(\frac{\Delta_{i}^{\bmod }-\Delta_{i}^{\exp }}{\sigma_{\Delta, i}^{\exp }}\right)^{2}\right],
$$

where $N$ is the number of $(\psi, \Delta)$ pairs and $M$ the number of variable parameters in the model. This quantity weighs each data point according to its experimental standard deviation $\sigma^{\text {exp }}$. The subscript $i$ can represent dependence on photon energy, time, and angle of incidence.

In order to model the dispersion (dependence of the dielectric function $\varepsilon$ on photon energy), a summation of oscillator terms was used. The dielectric function consists of the sum of a constant real term and contributions from two types of oscillators:

$$
\varepsilon(E)=\varepsilon_{1 \infty}+\varepsilon_{L}(E)+\varepsilon_{G}(E) .
$$

Absorptions at photon energies higher than the spectral range of interest cause $\varepsilon_{100}$ to be greater than unity. The Lorentzian term is

$$
\varepsilon_{L}(E)=\sum_{n=1}^{N_{L}} \frac{A_{n} E_{c n}^{2}}{E_{c n}^{2}-E^{2}-i B_{n} E},
$$

where $E_{c n}$ is the center energy of the $n$th oscillator, $A_{n}$ is its amplitude, and $B_{n}$ its broadening. The Gaussian term is more easily defined in terms of its imaginary part,

$$
\begin{aligned}
\varepsilon_{2 G}(E)= & \sum_{n=N_{L}+1}^{N_{L}+N_{G}} A_{n}\left\{\exp \left[-\left(\frac{E-E_{c n}}{B_{n}}\right)^{2}\right]\right. \\
& \left.+\exp \left[-\left(\frac{E+E_{c n}}{B_{n}}\right)^{2}\right]\right\},
\end{aligned}
$$

and noting that the real part $\varepsilon_{1 G}(E)$ is computed using the Kramers-Kronig relation. ${ }^{19}$ The number of Lorentzian and Gaussian oscillator terms are given by $N_{L}$ and $N_{G}$, respectively.

Optical anisotropy is induced by directional preference in the crystal orientation of the grains or the microstructure of a material. These preferences are manifested in the different dielectric responses depending on the electric-field direction of the electromagnetic wave. For symmetries which may be defined in terms of Cartesian coordinate directions, the relationship between the electric and displacement field vectors is expressed as ${ }^{20}$

$$
\mathbf{D}=\left(\begin{array}{c}
D_{x} \\
D_{y} \\
D_{z}
\end{array}\right)=\left[\begin{array}{ccc}
\varepsilon_{x} & 0 & 0 \\
0 & \varepsilon_{y} & 0 \\
0 & 0 & \varepsilon_{z}
\end{array}\right]\left(\begin{array}{c}
E_{x} \\
E_{y} \\
E_{z}
\end{array}\right)=\varepsilon \mathbf{E} .
$$

In this work, all directions parallel to the plane of the sample are equivalent, so two of the dielectric tensor elements will be equal (uniaxial symmetry). The $x$ direction is chosen as the intersection of the plane of incidence and the sample surface; $y$ is perpendicular to the plane of incidence. We will then refer to the "in-plane" dielectric function as $\varepsilon_{x y}\left(=\varepsilon_{x}\right.$ $=\varepsilon_{y}$ ), and the "out-of-plane" dielectric function will be $\varepsilon_{z}$.

Given the columnar nature of these pores, it is reasonable to expect that the layers would exhibit shape anisotropy which can be modeled by Bruggeman's effective medium approximation (EMA) ${ }^{5,21}$ This theory yields the effective dielectric function of a mixture from the dielectric functions of its components and their volume fractions. This approach has, in fact, been used to characterize porous alumina ${ }^{12,13}$ already, with perfect columnar anisotropy assumed in the model. However, ellipsometry can in many cases determine whether anisotropy is present and allow independent determination of the dielectric tensor elements $\varepsilon_{x y}$ and $\varepsilon_{z}$. Both methods are used here.

Bruggeman's EMA theory has also been generalized for cases with imperfect columnarity. ${ }^{22,23}$ Generally, electricfield components parallel to the plane of the sample surface will encounter boundaries between solid and solution or solid and void at varying angles; perpendicular components remain tangential to these boundaries, analogous to parallel capacitances. A direction-dependent depolarization factor $q$ is introduced to account for deviations from this behavior. For the case where all electric fields are perpendicular to boundaries, $q=1$; if all electrical fields are parallel to boundaries, $q=0$. Physical consistency requires that $q_{x}+q_{y}+q_{z}=1$. For isotropic material, $q_{x}=q_{y}=q_{z}=1 / 3$, while for columnar symmetry in the out-of-plane direction, $q_{z}=0$ and $q_{x}=q_{y}$ $=1 / 2$. The out-of-plane depolarization factor can then be used as a fit parameter for the general case of uniaxial shape anisotropy. This is an extension of the shape anisotropy model used previously for porous alumina.

\section{RESULTS}

\section{A. Monitoring of layer formation}

The anodization process was monitored at 15-s intervals by in situ multiwavelength SE. The instrument (M2000, J.A. Woollam Co., Inc.) collects 400 channels of data simultaneously corresponding to photon energies from 1.24 to 3.26 $\mathrm{eV}$. The wealth of information obtained during in situ ellipsometric monitoring can provide significant insight into process results. However, certain complications, especially the changing film properties during growth transitions, can make it difficult to determine these results unambiguously. In porous alumina formation, this problem is exacerbated in the early stages of growth, where a barrier layer of continuous alumina develops at the base of the porous layer, which is believed to actually decrease in thickness when pores begin forming, ${ }^{2}$ but eventually stabilizes at some steady-state thickness. In this study, the simplest model was employed 

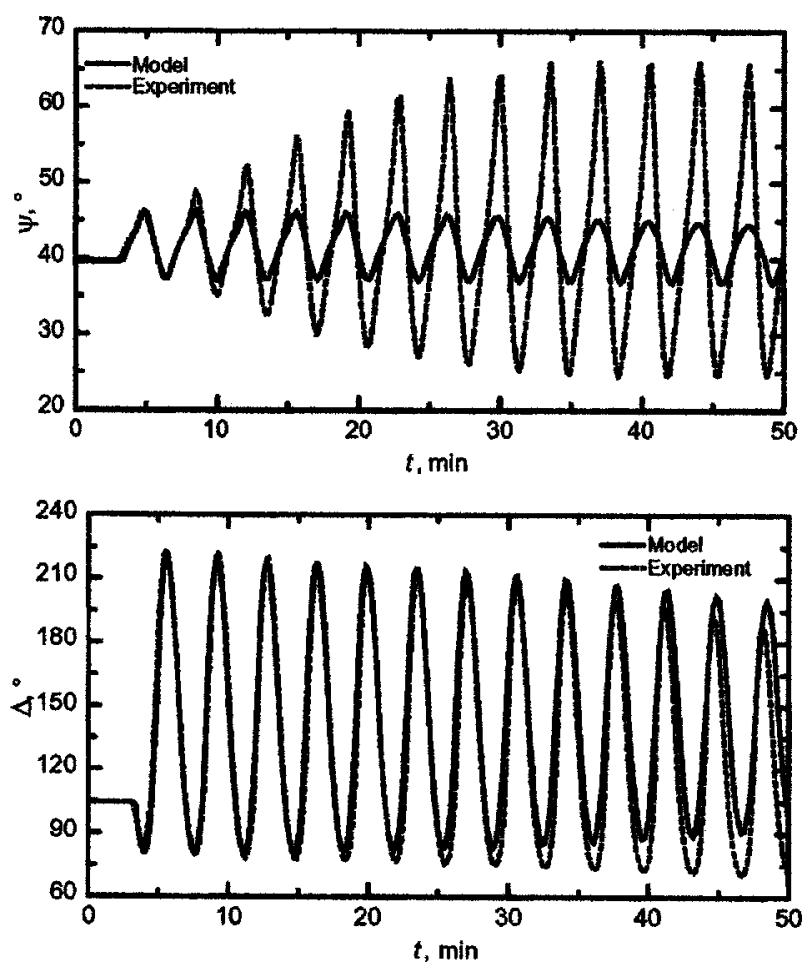

FIG. 3. Comparison of ellipsometric data at $1.75 \mathrm{eV}$ during layer formation to that of an isotropic model with no absorption or dispersion. This illustrates the deficiencies of the optical model when absorption and anisotropy are not included.

first to explain the results, and a series of alterations were then made to improve the agreement between the model and the generated data.

For the in situ analysis, the substrate (aluminum) dielectric function was determined at each photon energy by acquiring an initial measurement in solution and inverting the data with a regression fit. The dielectric function for the oxalic acid solution was determined using the thermal oxide silicon wafer referred to in the experimental section.

Figure 3 shows dynamic data at $1.75 \mathrm{eV}$ for $\psi$ and $\Delta$ during the alumina growth; other wavelengths exhibited similar characteristics. In the first few minutes of growth, the data may be modeled effectively using a transparent layer. As the process progresses, however, the data deviate from this model in a number of ways. The most prominent is the increasing amplitude of the $\psi$ oscillation, which is readily evident in the $\psi$ spectrum by the second oscillation. This is not accounted for by any physically consistent dispersion without including absorption in the spectral range being measured.

The initial fits were performed using representative photon energies: $1.24,1.75$, and $3.00 \mathrm{eV}$. This helped estimate dispersion parameters so later fits involving all wavelengths could be initialized near a probable solution. A modeling program was used to add dielectric function terms systematically while maintaining Kramers-Kronig consistency. Obvious deficiencies are present in the initial model fit shown in Fig. 3, which used a single index value with no dispersion or absorption, resulting in a MSE of 261. This quantity is not useful in an absolute sense, but does indicate comparative fit quality between models involving the same data set. Disper-
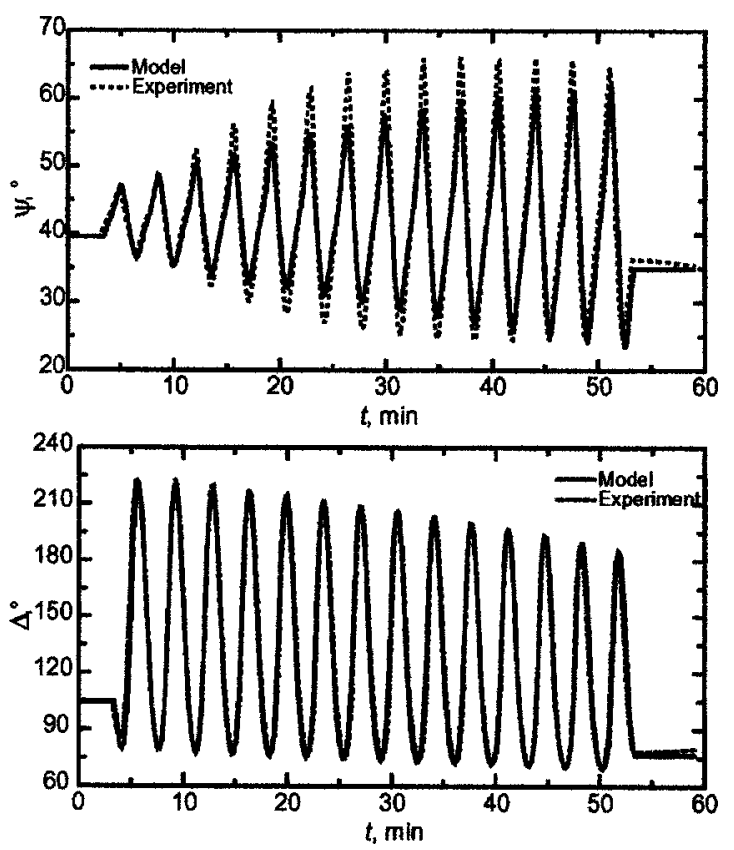

FIG. 4. Fits to experimental data at $1.75 \mathrm{eV}$ for the entire deposition after modeling using method 1 for all wavelengths.

sion in the real part of the dielectric function alone was included by addition of a zero-broadening Lorentzian oscillator ("pole") in the ultraviolet (UV) at $11 \mathrm{eV}$, which reduced the MSE to 192. According to the Kramers-Kronig relations, absorption in one spectral range is always associated with dispersion over a broader range. The pole accounts for this dispersion in the visible range due to UV absorption that is outside the spectral range of the instrument. The primary qualitative effect of isotropic index dispersion, as might be expected, was to simultaneously match the oscillation periods more uniformly over the spectrum. The increasing $\psi$ oscillation amplitude with time and photon energy was
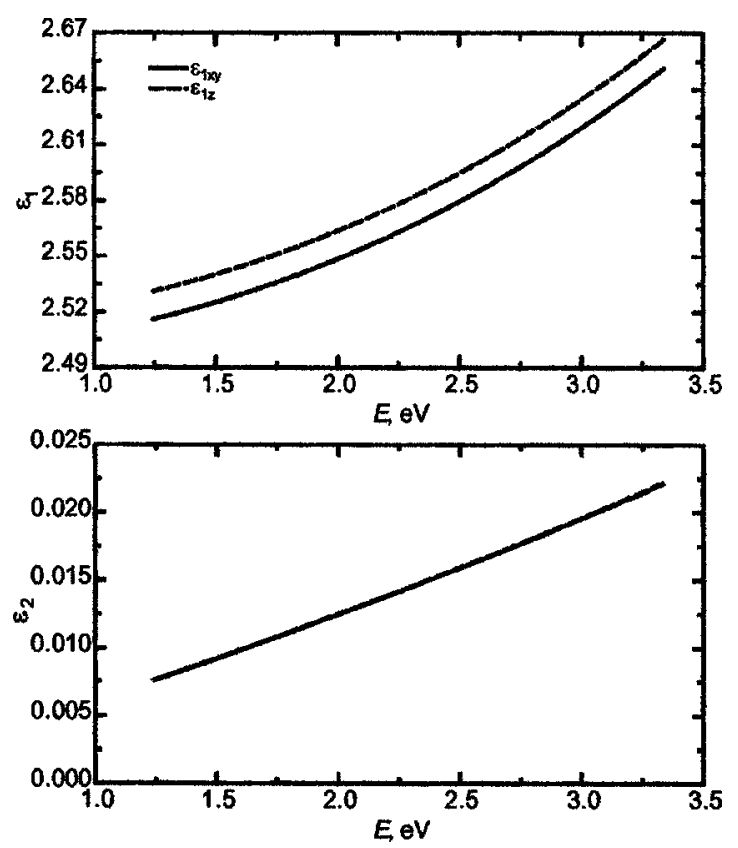

FIG. 5. Dielectric functions based on method 1 analysis of in situ data during layer formation. 


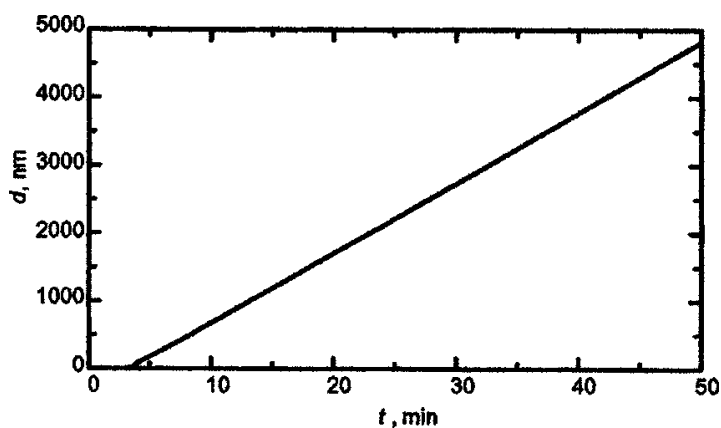

FIG. 6. Thickness of porous alumina layer as a function of time during layer formation

largely accounted for by adding a Lorentz oscillator in the UV range at about $15 \mathrm{eV}$, due to the associated absorption tail, which yielded a MSE of 148 . The final isotropic model used four fit parameters: $\varepsilon_{1 \infty}$, pole magnitude, oscillator amplitude, and broadening.

Without inclusion of anisotropy, the decreasing average $\Delta$ value with time still could not be accounted for. Allowing $\varepsilon_{1 \infty}$ and pole magnitude for the out-of-plane dielectric function to differ from the in-plane values reduced the MSE to 110. Adding thickness nonuniformity to the isotropic model at this point did not have as strong an effect as the inclusion of anisotropy in the model. Absorption anisotropy was tried in the form of an UV Lorentz oscillator, but did not produce a significantly better fit than anisotropy in $\varepsilon_{1}$ only. Finally, thickness nonuniformity was incorporated into the model based on the reduced oscillation amplitude especially during the final 15 min of growth, reducing the MSE to 89 . The fit, shown in Fig. 4, indicated a thickness nonuniformity of 3\%. In general, thickness nonuniformity in the model caused more attenuation of interference oscillations in the UV than was observed experimentally. This could mean that other
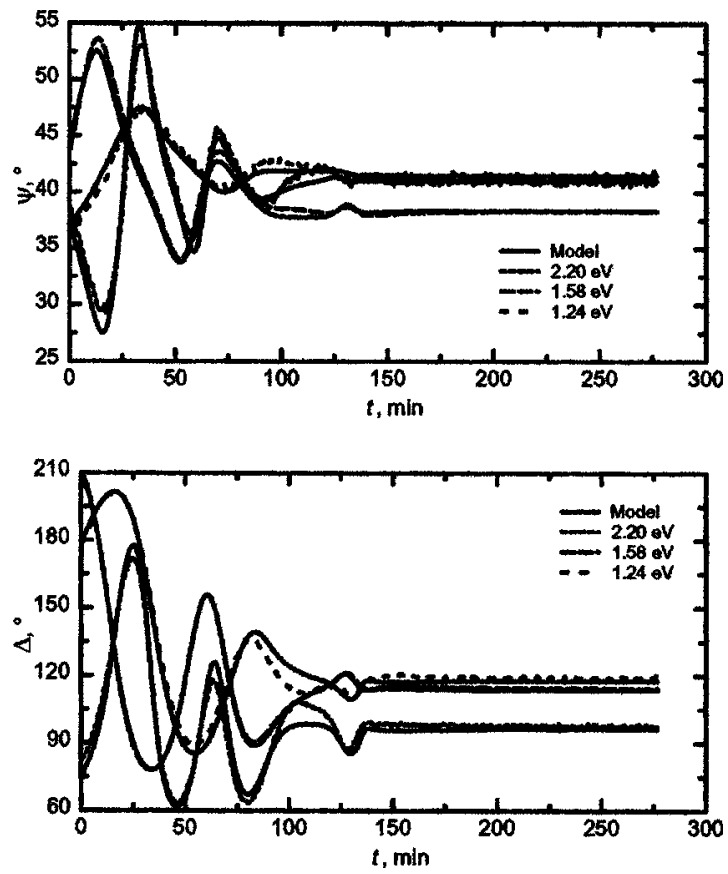

FIG. 7. Time-dependent fits to ellipsometric parameters during chemical etching.

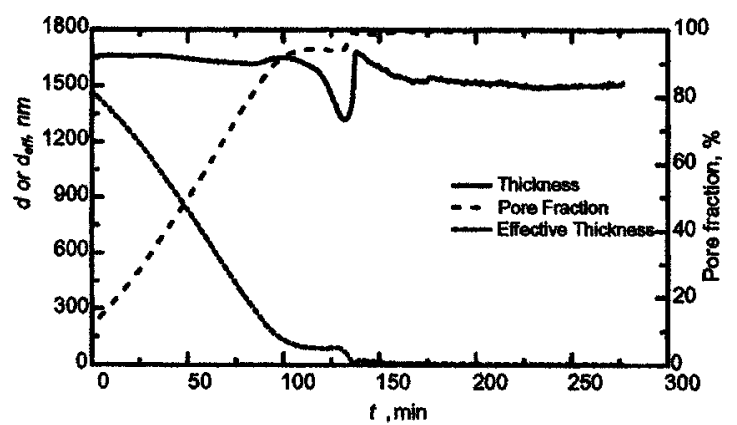

FIG. 8. Result of time-dependent fits during chemical etching of porous alumina.

nonidealities, such as changing film properties during growth, may have contributed to the data with a similar effect. These effects would be difficult to distinguish, however, from the data acquired.

The preceding approach to fitting the data, fitting for both the in-plane and out-of-plane dielectric functions, will be referred to as method 1 . Method 2 was to fit for the dielectric function of the solid fraction and use a Bruggeman EMA to introduce optical anisotropy. The dielectric function of the porous alumina solid fraction is obtained by starting with the in-plane dielectric function from method 1 and fitting only the amplitudes and $\varepsilon_{100}$ to an isotropic model. The volume fraction of liquid (void for dry samples) is introduced as an additional fit parameter, assuming $q_{z}=0$. For in situ data, the same dielectric function that was used previously for the ambient was used for the liquid fraction of the porous layer.

The modeling software can also vary the depolarization factor $q_{z}$ while maintaining the appropriate constraints for $q_{x y}$. The final step is to fit all the solid fraction dielectric function parameters, $q_{z}$, and $r$ simultaneously. The number of parameters for the solid fraction in method 2 is the same as that for the in-plane dielectric function in method 1. The resulting method 2 MSE was slightly higher $(\sim 5 \%)$, while the resulting void fraction of 0.05 was considerably lower than the estimated value from the atomic force microscopy in Fig. 1. With significant correlation between the effects of $q_{z}$ and $r$, the best fit was obtained for $q_{z}=0.05$.

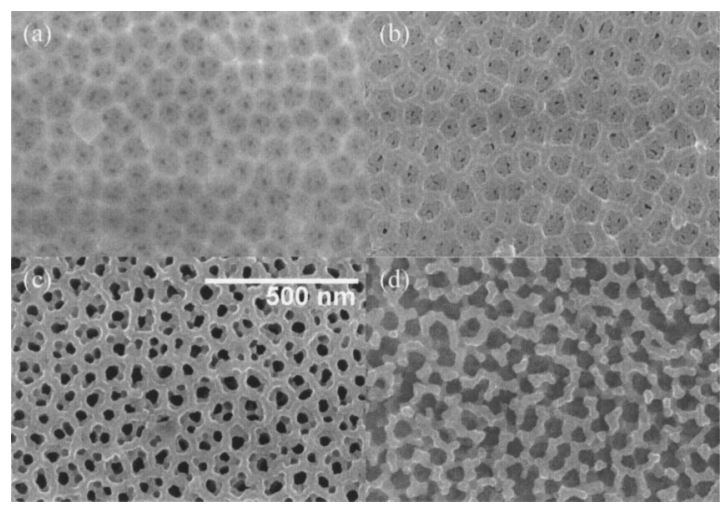

FIG. 9. Scanning electron micrographs of chemical etch effects. The conditions are (a) as-anodized, (b) after 15-min etching, (c) after $45 \mathrm{~min}$, and (d) after $90 \mathrm{~min}$ 

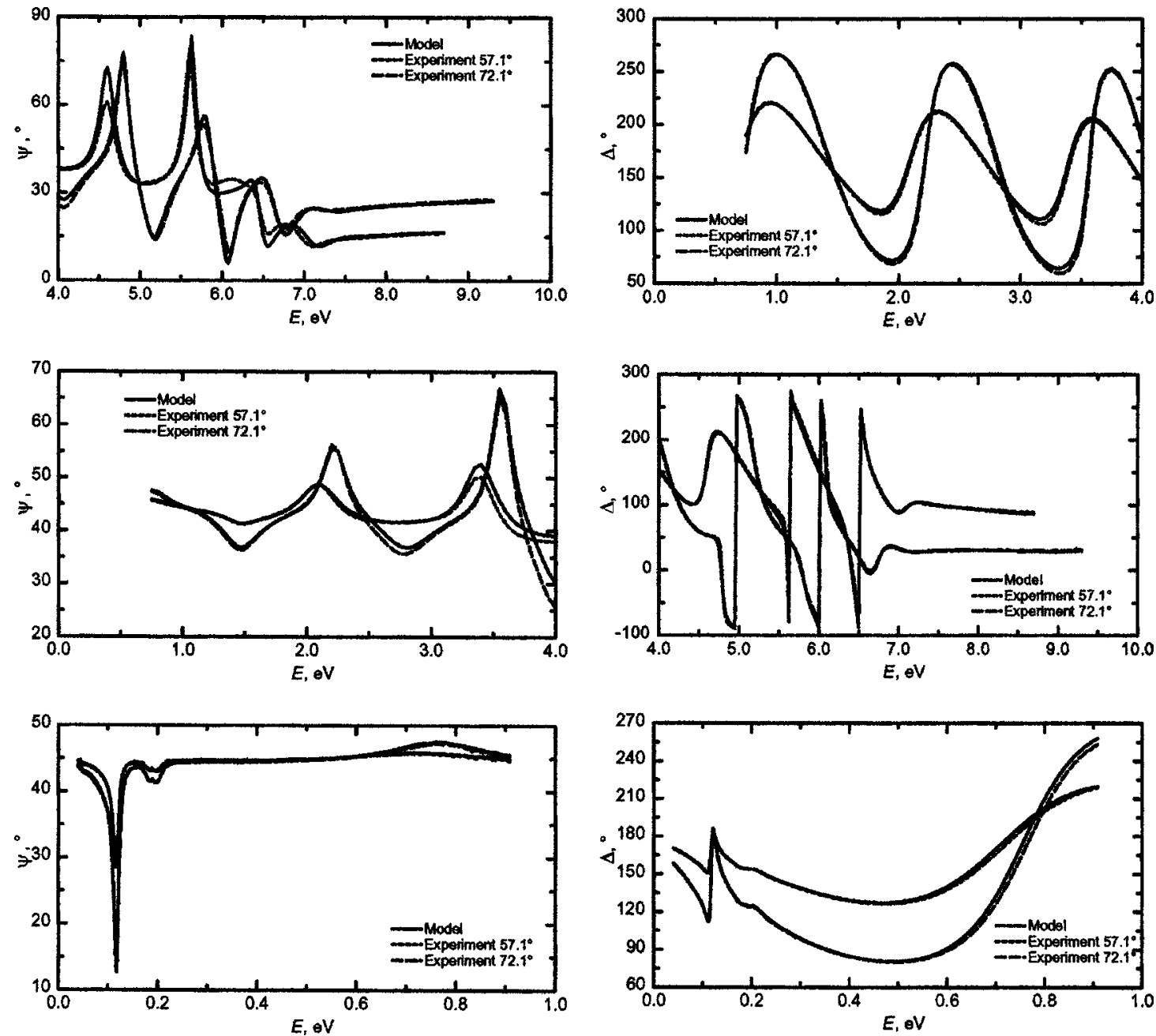

FIG. 10. Fits to ellipsometric data for a dry porous alumina layer. The thickness was fit to $316 \mathrm{~nm}$ with a 17 -nm roughness layer.

The resulting values of $\varepsilon$ for the solid (2.65 at $E$ $=3.1 \mathrm{eV})$ are somewhat lower than previously published values for bulk alumina (3.19 and 3.16 for $\varepsilon_{x y}$ and $\varepsilon_{z}$, respectively). ${ }^{24}$ This is similar to the result of Kooij et al., who assumed $q_{z}=0$ in an ex situ study of porous alumina formed in sulfuric acid. ${ }^{12}$ They attributed this to a $28 \%$ "nanoporosity" caused by extremely small-scale voids within the solid fraction of the material. They did not include the effect of absorption in their work.

Figures 4 and 5 show the final ellipsometric fits and dielectric functions obtained using method 1 . The results from the two methods were actually very similar, with the index anisotropy $\Delta \varepsilon=\varepsilon_{z}-\varepsilon_{x y} \cong 0.025$ in both cases. The magnitude of the index using the EMA was generally $\sim 0.022$ lower, with a correspondingly higher $(<1 \%)$ indicated growth rate. The absorption in the EMA case was not substantially different.

After the best dielectric function spectra were determined, the linearity of the growth was checked by fitting each spectrum for layer thickness only. The results are shown in Fig. 6, indicating a highly linear growth. The average growth rate for this sample was $103 \mathrm{~nm} / \mathrm{min}$.

\section{B. Monitoring of layer removal}

In situ SE was also used to model the chemical etching process to assess when the entire porous layer was removed. These data were also analyzed to determine the time dependence of the etch process. The spectral range was limited because of the absorption of the etch solution, which is absorbing at photon energies above $2.2 \mathrm{eV}$. A spectrum in the early stages of etching was fit to obtain the dielectric function for the solid fraction, layer thickness, and void percentage, with the best fit found for $q_{z}=0$. The latter was held constant for the time-dependent fit, for which layer thickness and void percentage were the only parameters. Only method 2 was utilized. Figure 7 shows data and fit during etching. The resulting layer thickness and pore fraction are shown in Fig. 8. Effective thickness is the layer thickness from the fit multiplied by the solid fraction. The fit suggests that the layer underwent very little change in thickness, but became progressively more porous due to etching. This is consistent with an isotropic etch which affects the inside walls of the pores. The fit quality deteriorated when the layer porosity exceeded $80 \%$, and the fit parameter behavior became somewhat erratic, indicating that the low solid fraction was probably insufficient to maintain a continuous layer. At this stage 
areas of porous material, a few microns across separated by cracks less than a micron in width, were observed with scanning electron microscopy (SEM). The layer thickness became indeterminate once the void fraction approached $100 \%$.

Images from SEM during the various stages of the process are shown in Fig. 9. These show further evidence of the enlargement of the pores during chemical etching. They also show a top layer approximately $50 \mathrm{~nm}$ thick which has a higher void fraction than the bulk. This was treated as a roughness layer for the succeeding analysis of dried layers.

\section{Wide spectrum results for dried layers}

The goal of the ex situ analysis was to obtain a unified dispersion model which describes the behavior of the porous alumina over the entire photon energy range from 0.04 to 9.3 $\mathrm{eV}$. Since most of the insight into the dielectric function of porous alumina comes from the spectral dependence, data from only two angles of incidence $\left(57.1^{\circ}\right.$ and $\left.72.1^{\circ}\right)$ were used in the ex situ study. The visible region was fit first using a dielectric function similar to that of the in situ analysis, resulting in an estimate for the film thickness. When the UV data were introduced, the absorptions in that range could be determined in more detail. A starting point for the dielectric function of the porous layer was obtained by fitting at each photon energy assuming no anisotropy and the thickness obtained in the visible region. This photon energy dependence was then approximated by Gaussian and Lorentzian oscillators, and the fit mode was changed to vary the parameters of the oscillators rather than the dielectric function at each wavelength. With the inclusion of two Gaussian oscillators centered at 11.59 and $4.71 \mathrm{eV}, \varepsilon_{1 \infty}$ could be set to unity and the pole at $11 \mathrm{eV}$ could be eliminated.

Since the transparent region extends to about $0.3 \mathrm{eV}$, the addition of infrared-absorption peaks has no effect on the visible/UV analysis. The major ellipsometric feature in the infrared at $0.11 \mathrm{eV}$ (Fig. 10) is due to the longitudinal optical mode [point where $\varepsilon_{1}(E)$ passes through 0 ] associated with an absorption peak centered at $0.0812 \mathrm{eV}\left(655 \mathrm{~cm}^{-1}\right)$. The dispersion nearby and at lower photon energy is best modeled by two overlapping Gaussian oscillators, depicted in Fig. 11(a) and shown in Table I as 5G and 6G. These appear to be related to major absorption peaks seen by others in polycrystalline alumina. ${ }^{25}$ Related peaks in crystalline alumina have also been identified. ${ }^{26}$ The doublet between 0.16 and $0.22 \mathrm{eV}$ could be related to the incorporation of some component of the anodization solution. This feature was fit by Lorentzian line shapes better than by Gaussian line shapes.

After the best isotropic model was found, anisotropy was added to the model. As in the in situ case, both methods 1 and 2 were used. The anisotropy in method 1 consisted of only two parameters: the amplitudes of the UV Gaussian oscillator terms. This provided a $10 \%$ advantage in MSE over method 2, for which $q_{z}$ tended toward 0 , effectively leaving only one fit parameter, $r$, to adjust anisotropy. Fits shown in Fig. 10 are for method 1, but those for method 2 look essentially the same. For this reason the dielectric function anisotropy shape was constrained to look similar to that (a)

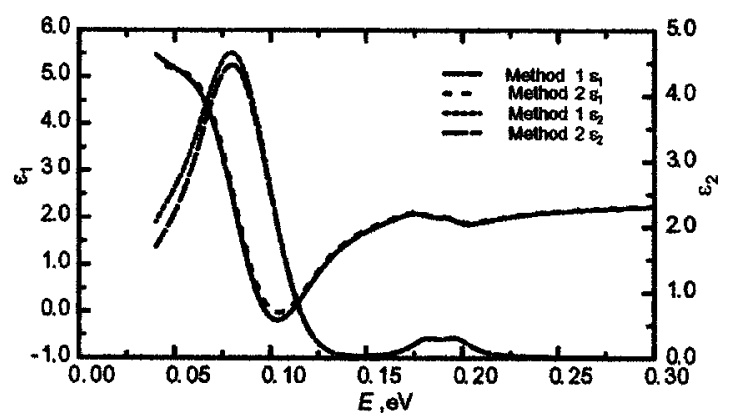

(b)

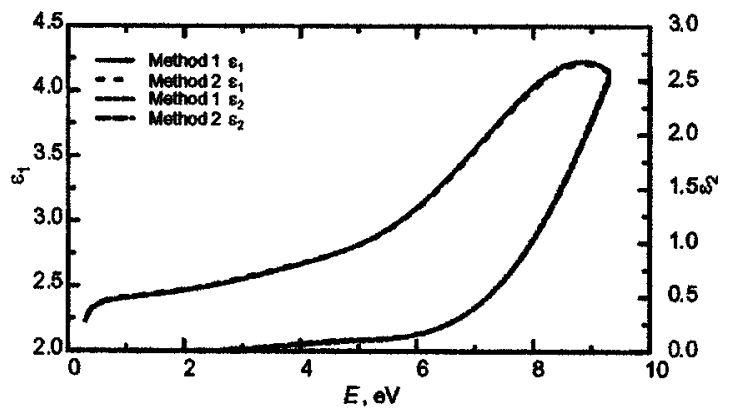

(c)

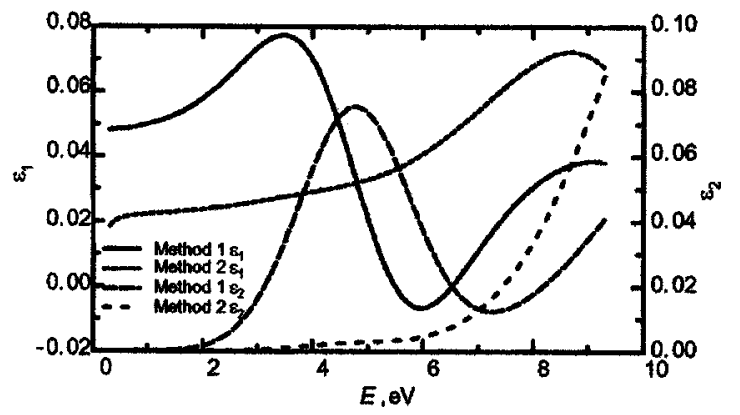

FIG. 11. Dielectric functions for dry porous alumina. [(a) and (b)] In-plane dielectric function for methods 1 and 2. (c) Anisotropy in the dielectric function. Here $\Delta \varepsilon=\varepsilon_{\mathrm{z}}-\varepsilon_{\mathrm{xy}}$.

of the solid alumina. The difference in MSE is primarily seen in the $2 \mathrm{G}$ oscillator feature at $4.7 \mathrm{eV}$, which has a much larger relative magnitude in the anisotropy. The present study does not contain evidence that would either prove or disprove this feature, since it is conceivable that the material growth also induces anisotropy within the solid fraction. The Bruggeman EMA model tended in this case to a pure colum-

TABLE I. Final oscillator parameters for dried porous alumina dielectric function using method 1. Terms are marked $\mathrm{G}$ for Gaussian and $\mathrm{L}$ for Lorentzian. Terms 7 and 8 are the anisotropy terms.

\begin{tabular}{cccc}
\hline \hline Oscillator & $\begin{array}{c}\text { Center energy } \\
(\mathrm{eV})\end{array}$ & Amplitude & $\begin{array}{c}\text { Broadening } \\
(\mathrm{eV})\end{array}$ \\
\hline 1G $\left(\varepsilon_{x y}\right)$ & 11.59 & 4.68 & 4.88 \\
$2 \mathrm{G}\left(\varepsilon_{x y}\right)$ & 4.71 & 0.0688 & 2.37 \\
$3 \mathrm{~L}\left(\varepsilon_{x y}\right)$ & 0.120 & 0.242 & 0.0205 \\
$4 \mathrm{~L}\left(\varepsilon_{x y}\right)$ & 0.1811 & 0.224 & 0.0195 \\
$5 \mathrm{G}\left(\varepsilon_{x y}\right)$ & 0.0425 & 1.778 & 0.0420 \\
$6 \mathrm{G}\left(\varepsilon_{x y}\right)$ & 0.0812 & 4.47 & 0.0420 \\
$7 \mathrm{G}(\Delta \varepsilon)$ & 11.59 & 0.0715 & 4.88 \\
$8 \mathrm{G}(\Delta \varepsilon)$ & 4.71 & 0.0750 & 2.37 \\
\hline
\end{tabular}




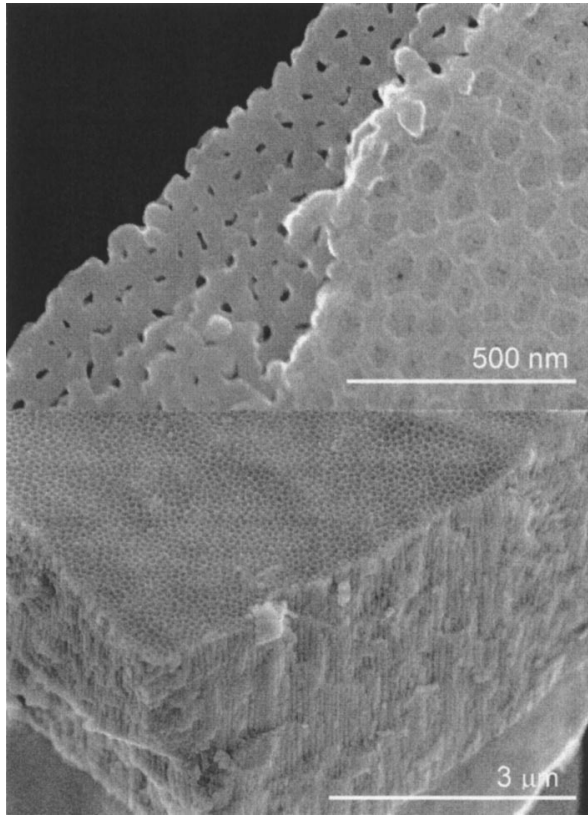

FIG. 12. SEM micrographs of cleaved edges of porous alumina to illustrate the characteristics of the interior of the material.

nar structure $\left(q_{z}=0\right)$, and has the advantage that its physical consistency is not in question.

Error bars on $q_{z}$, however, indicated that it was not well determined. To investigate this, fits were performed at 11 different values of out-of-plane depolarization factors varying from 0 (perfect columnar anisotropy) to 0.33 (isotropic). The resulting MSEs did not vary strongly for thinner $(\sim 1.5 \mu \mathrm{m})$ samples, indicating that stronger anisotropy is not easily distinguishable from a larger void fraction in that case. The best MSE was found for $q_{z}=0.05$ corresponding to a void fraction of about $5 \%$. Though AFM would indicate a larger void fraction at the surface, SEM of a cleaved edge in Fig. 12 shows the bulk probably has a significantly smaller void fraction than the top surface. This also shows imperfections in the columnar grains when viewed from the side, supporting the idea that $q_{z}>0$. Attempts to remove this top surface using the oxide stripping solution resulted in enlarged pores in the material, as shown in Fig. 9. The final fit and resulting dielectric functions are shown in Figs. 10 and 11. Oscillator parameters for the full spectrum for each method are in Tables I and II.

TABLE II. Final oscillator parameters for dried porous alumina solid fraction dielectric function using method 2. Terms are marked $\mathrm{G}$ for Gaussian and $\mathrm{L}$ for Lorentzian.

\begin{tabular}{cccc}
\hline \hline Oscillator & $\begin{array}{c}\text { Center energy } \\
(\mathrm{eV})\end{array}$ & Amplitude & $\begin{array}{c}\text { Broadening } \\
(\mathrm{eV})\end{array}$ \\
\hline $1 \mathrm{G}$ & 11.59 & 4.940 & 4.88 \\
$2 \mathrm{G}$ & 4.71 & 0.0862 & 2.37 \\
$3 \mathrm{~L}$ & 0.197 & 0.255 & 0.0205 \\
$4 \mathrm{~L}$ & 0.1811 & 0.241 & 0.01953 \\
$5 \mathrm{G}$ & 0.0425 & 1.500 & 0.0420 \\
$6 \mathrm{G}$ & 0.0812 & 4.64 & 0.0420 \\
\hline \hline
\end{tabular}

\section{DISCUSSION AND CONCLUSION}

Porous alumina films were grown from evaporated aluminum on silicon and characterized with in situ visible SE, ex situ SE over the spectral range of 0.030 to $9.3 \mathrm{eV}$, SEM, and AFM. Optical dispersion models were used to maintain physical consistency for the dielectric function dependence on photon energy. The in situ analysis indicated homogeneous growth with a constant growth rate of $103 \mathrm{~nm} / \mathrm{min}$, and that subsequent chemical etching of the layers primarily removes material from inside the pores. Fitting for the outof-plane depolarization factor in an anisotropic Bruggeman effective medium approximation indicated that growth was generally, but not perfectly, columnar. As-grown layers were found to have void fractions in the 5\% range, as determined by SE.

Two methods were used to model the anisotropy in the films. Fitting for in- and out-of-plane dielectric functions independently (method 1) allowed for anisotropy within the solid itself. In dried samples this yielded different shapes for $\varepsilon_{x y}$ and $\varepsilon_{z}$ in the UV, but this is not fully conclusive without the ability to monitor growth through the absorbing oxalic acid solution. Using an isotropic solid fraction and purely shape-induced anisotropy (method 2) yielded slightly lower fit quality. The dielectric function of the solid fraction of these layers was near published values for bulk alumina in the visible range. The best-fit optical dispersion model parameters are presented in tabular form. These results were corroborated by scanning electron microscopy, which also indicated the presence of a highly porous initial growth (top) layer with a less-ordered, less-porous layer beneath. The effects of anisotropy are generally consistent with previous work which assumed columnar geometry.

\section{ACKNOWLEDGMENT}

The authors would like to extend special thanks to Dr. Kit Lee of the University of Nebraska Microscopy Core Facility for the SEM images.

${ }^{1}$ O. Jessensky, F. Muller, and U. Gosele, Appl. Phys. Lett. 72, 1173 (1998).

${ }^{2}$ L. Menon, Quantum Dots and Nanowires, edited by S. Bandyopadhyay and H. S. Nalwa(American Scientific, 2003, Stevenson Ranch, CA, 2003), p.141.

${ }^{3}$ S. Polarouthu, M.S. thesis, University of Nebraska-Lincoln, 2003.

${ }^{4}$ P. Kaipa, M.S. thesis, University of Nebraska-Lincoln, 2004.

${ }^{5}$ H. Arwin, L. M. Karlsson, A. Kozarcanin, D. W. Thompson, T. Tiwald, and J. A. Woollam ,Phys. Status Solidi C (accepted).

${ }^{6}$ B. E. Collins, K. P. S. Dancil, G. Abbi, and M. J. Sailor, Adv. Funct. Mater. 12, 187 (2002).

${ }^{7}$ L. M. Karlsson, P. Tengvall, I. Lundstrom, and H. Arwin, Phys. Status Solidi A 197, 326 (2003).

${ }^{8}$ L. M. Karlsson, R. Tengvall, I. Lundstrom, and H. Arwin, J. Colloid Interface Sci. 266, 40 (2003).

${ }^{9}$ S. Kodera et al., Anal. Biochem. 321, 65 (2003).

${ }^{10}$ I. N. Lees, H. H. Lin, C. A. Canaria, C. Gurtner, M. J. Sailor, and G. M. Miskelly, Langmuir 19, 9812 (2003).

${ }^{11}$ S. Zangooie, R. Bjorklund, and H. Arwin, Thin Solid Films 313, 825 (1998).

${ }^{12}$ E. S. Kooij, H. Wormeester, A. C. Galca, and B. Poelsema, Electrochem. Solid-State Lett. 6, B52 (2003).

${ }^{13}$ A. C. Galca, E. S. Kooij, H. Wormeester, C. Salm, V. Leca, J. H. Rector, and B. Poelsema, J. Appl. Phys. 94, 4296 (2003).

${ }^{14}$ S. L. Pan and L. J. Rothberg, Nano Lett. 3, 811 (2003).

${ }^{15}$ Guide to Using WVASE32 (Software Manual) (J.A. Woollam Co, Inc., Lin- 
coln NE, 2003).

${ }^{16}$ S. Bandyopadhyay et al., Nanotechnology 7, 360 (1996).

${ }^{17}$ R. M. A. Azzam and N. M. Bashara, Ellipsometry and Polarized light (North-Holland, New York, NY, 1977).

${ }^{18}$ J. A. Woollam, B. Johs, C. M. Herzinger, J. Hilfiker, R. Synowicki, and C. L. Bungay, Optical Metrology, in Critical Reviews of Optical Science and Technology Vol. CR72 (SPIE, Bellingham, WA, 1999.

${ }^{19}$ F. Wooten, Optical Properties of Solids (Academic, New York, 1972).

${ }^{20} \mathrm{P}$. Yeh, Optical Waves in Layered Media (Wiley, New York, 1988).
${ }^{21}$ D. A. G. Bruggeman, Ann. Phys. 24, 636 (1935)

${ }^{22}$ D. A. G. Bruggeman, Ann. Phys. 25, 645 (1936).

${ }^{23}$ A. Heilmann, Polymer Films with Embedded Metal Nanoparticles (Springer, Berlin, 2003).

${ }^{24}$ M. Bass and Optical Society of America, Handbook of Optics, 2nd ed. (McGraw-Hill, New York, 1995).

${ }^{25}$ C. A. Worrell, J. Mater. Sci. 21, 781 (1986).

${ }^{26}$ M. Schubert, T. E. Tiwald, and C. M. Herzinger, Phys. Rev. B 61, 8187 (2000). 